The Psychological Record, 2010, 60, 337-354

\title{
CONSEQUENCE VALUING AS OPERATION AND PROCESS: A PARSIMONIOUS ANALYSIS OF MOTIVATION
}

\author{
Robert Whelan \\ Trinity College Dublin \\ Dermot Barnes-Holmes \\ National University of Ireland, Maynooth
}

\begin{abstract}
The concept of the motivating operation (MO) has been subject to 3 criticisms: (a) the terms and concepts employed do not always overlap with traditional behavior-analytic verbal practices; (b) the dual nature of the MO is unclear; and (c) there is a lack of adequate contact with empirical data. We offer a more parsimonious approach to motivation, extending Skinner's $(1938,1953)$ early work on this topic, and introduce two new key terms: consequence-valuing operation (CVO) and consequence-valuing process (CVP). Our account focuses on the value of the reinforcer in terms of the level of responding that occurs relative to some previously measured baseline. We suggest that the concepts of the CVO and CVP will facilitate the identification, analysis, and manipulation of motivational factors in the treatment of behavior disorders.
\end{abstract}

Key words: motivation, establishing operation, motivating operation, consequence-valuing operation, consequence-valuing process

In the words of Catania (1992), "When we study motivation or drive we're concerned with what makes consequences more or less effective [emphasis added] as reinforcers or as punishers" (p. 77). Keller and Schoenfeld (1950) introduced the term establishing operation (EO; p. 269) to distinguish motivational effects from reinforcement effects. Skinner (1953) distinguished between satiation and deprivation and other types of environmental variables that influence the effectiveness of reinforcers. For example, for a water-deprived person "a large group of operants are strengthened" (p. 143), such as going to the kitchen, operating a drinking fountain, and so on. Leigland (1984), however, noted that the treatment of motivational variables in behavior analysis has suffered from the lack of a clear functional definition.

Michael (1982, 1988, 1993a, 1993b, 2000) and his colleagues (Laraway, Snycerski, Michael, \& Poling, 2001/2002, 2003) have sought to more clearly describe motivational variables by developing and extending Skinner's treatment of motivation, most recently by introducing the term motivating

Correspondence concerning this article should be addressed to Robert Whelan, Trinity Centre for Bioengineering, Trinity College Dublin, Dublin 2, Ireland. E-mail: robert.whelan@tcd.ie 
operation (MO). Michael's approach has received widespread attention and has been cited in numerous articles in the past 25 years. There is no doubt that Michael's analysis has played an important role in orientating behavior analysts toward the important role of motivational factors in influencing behavior. Indeed, the Journal of Applied Behavior Analysis has featured several empirical and theoretical papers on the topic of EOs/MOs (see McGill, 1999; Michael, 2000; Smith \& Iwata, 1997). This research has shown that the manipulation of motivational factors is a powerful method for reducing problem behavior and for increasing desired behavior (Iwata, Smith, \& Michael, 2000). The analysis of motivational variables has, however, received comparatively little attention in the basic behavior-analytic literature (but see McPherson \& Osborne, 1988, for a basic-research example).

The importance of motivational variables calls for a precise and coherent conceptual analysis. However, it is important to recognize that any new concept in our science should be scrutinized carefully before that concept is widely accepted. It is on this basis, perhaps, that the MO concept has not gone entirely unchallenged (e.g., Catania, 1993; Cherpas, 1993; McDevitt \& Fantino, 1993). Our aim in the current article is to briefly consider the challenge to the MO and to present an alternative account of motivation. Our approach is strongly influenced by the fact that the scientific method is biased toward parsimony and that this bias has served behavior analysis well in constructing an account of nonhuman and human psychology (Chiesa, 1994). We believe that a relatively straightforward explanatory framework for describing motivated behavior is possible. And we invite others to decide for themselves if our treatment provides an adequate and parsimonious explanatory framework in which to interpret motivational factors.

\section{Motivating Operations}

Borrowing from Keller and Schoenfeld (1950), Michael (1982, 1993a) initially proposed the concept of the EO, and Michael and colleagues more recently offered the concept of the MO (Laraway et al., 2003). The MO incorporates both the EO and the EO's negative counterpart, the abolishing operation (AO). Laraway et al. defined the MO as some environmental variable (event, operation, condition, etc.) that, first, alters the consequential effectiveness of other events, objects, or stimuli. Laraway et al. described this as the value-altering effect, which is a generic description of a change in the effectiveness of any operant consequence. Second, Laraway et al. suggested that an MO changes the current frequency of all behavior that has been reinforced by those events, objects, or stimuli and described this as the behavior-altering effect. Laraway et al. recommended that behavior analysts "(a) use the verb evoke to describe an increase and the verb abate to describe a decrease in responding due to the action of antecedents and (b) denote the former an evocative effect and the latter an abative effect" (p. 411). EOs for reinforcers have evocative effects, as do AOs for punishers. AOs for reinforcers have abative effects, as do EOs for punishers. For example, according to the explanatory framework described in Laraway et al., food deprivation is an MO that increases the reinforcing effect of food and also evokes behaviors that have in the past led to food. Food satiation is an AO that decreases the reinforcing effect of food and abates behaviors that have in the past led to food. 
In previous publications, Michael (1993a) introduced the term unconditioned establishing operations (UEOs). In this case, the reinforcerestablishing effect is unlearned and a consequence of the evolution of the species. More specifically, UEOs are described as environmental events, operations, or stimulus conditions that regulate the momentary effectiveness of unconditioned reinforcers (and also the momentary effectiveness of the conditioned reinforcers based on those unconditioned reinforcers). Examples of UEO's include food, water, activity, sleep, temperature changes, sexual reinforcers, and painful stimulation in the contexts of escape and aggression. The concept of establishing stimuli $\left(\mathrm{S}^{\mathrm{E}} \mathrm{S}\right)$, first mentioned in Michael (1982), was renamed in Michael (1993a) as conditioned establishing operations (CEOs). As with $S^{\mathrm{E}}$, CEOs were hypothesized as variables that alter the reinforcing effectiveness of other events as a result of an individual organism's history. Three types of CEOs were described: a surrogate CEO, which involves correlating a stimulus with a UEO; a reflexive CEO, which involves correlating a stimulus with worsening or improvement; and a transitive CEO, which is described as conditional conditioned reinforcement and punishment (see Michael, 1993a, pp. 198-205, for a comprehensive treatment of these concepts). We discuss these subdivisions in detail at a later stage.

\section{Problems with Motivating Operations}

Although behavior analysts have recognized that dealing with the issue of motivation is important, and Michael and his colleagues have clearly attempted to do this, some have questioned the treatment they have offered of this important topic (e.g., Catania, 1993; McDevitt \& Fantino, 1993). The main criticisms are based on the introduction of several new terms and concepts that do not always overlap with established verbal practice, concern over the dual nature of the MO, and a lack of adequate contact with empirical data. We briefly review these three criticisms before suggesting a possible alternative.

\section{Terminological Ambiguities}

At the present time, the following key terms have been offered by Michael and his colleagues in their treatment of motivation: motivating operation (MO) and its two subtypes, establishing operation (EO) and abolishing operation (AO). The establishing operation has two subtypes, the unconditioned establishing operation (UEO) and the conditioned establishing operation (CEO). The latter has itself three subtypes, the surrogate CEO, the reflexive $\mathrm{CEO}$, and the transitive CEO. A number of other terms are also used to describe the effects of the operations outlined above, for example, the evocative and the abative effects (e.g., Laraway et al., 2003). Moreover, other terms have also been introduced that are not directly related to motivationunconditioned elicitor (UE), conditioned elicitor (CE), and unconditioned conditioner (UC; Michael, 1983)-but nonetheless appear to be important for Michael's conceptual analysis. The proliferation of so many new terms has been criticized by others in the field, such as Catania (1993), who commented that "Michael's article introduces many terms and abbreviations without justification. ... I believe that introducing so many new ones wholesale can only lead to terminological confusion" (p. 219). 
Some confusion may also arise from the use of terms in ways that depart from traditional behavior-analytic verbal practices. In constructing functional analytic treatments of behavior, it has proved useful to distinguish between behavioral operations and behavioral processes. Operations refer to the reinforcement contingencies and other relations between organism and environment that the researcher or practitioner arranges. Processes refer to the behavioral changes that occur as a result of the operations. So, for example, a researcher might establish reinforcement as an operation by arranging a contingency between a rat's lever pressing and the delivery of food, and then subsequently observe reinforcement as a process if the animal's lever pressing increases as a function of the operation. Consistent with this distinction between behavioral operation and process, Catania (1993) argued that the term establishing operation is inherently misleading. As he pointed out, operations are typically defined as manipulations of the environment, and thus, the modifiers unconditioned and conditioned are not appropriate for the term establishing operation. Only the reinforcing properties of particular stimuli can be learned or unlearned (i.e., a behavioral process); the manipulations of the operations themselves are not learned behaviors. In addition, Catania pointed out that the terms unconditioned and conditioned, in the context of UEO and CEO, are overly restrictive, because "conditioned" does not encompass all learned behaviors. For example, the imprinting of a duckling on a specific stimulus (typically a parent) is not a UEO, but neither is it a consequence of conditioning. Catania suggested that the terms learned and unlearned may substitute more satisfactorily.

\section{The Dual Nature of the MO}

Michael's definition of the MO involves a dual-process concept, in that a distinction is made between value-altering and behavior-altering effects. According to Michael, Hixson, and Clark (1997), this distinction is made for the following reason:

We can't have our organism engaging in behavior that has made it warmer when it is actually food deprived, and vice versa. And because real organisms show these differential forms of behavior prior [emphasis added] to actually achieving the relevant reinforcement-during extinction responding, for example-the differential moment-by-moment behavioral frequency cannot depend upon the organism making contact with the reinforcer. (pp. 240-241)

Michael (2000) expanded further on the nature of the EO/evocative relation and again cautioned against conceptualizing the evocative effect of the EO as a product of the organism's contact with the more effective reinforcement. According to Michael,

food deprivation makes food a more effective form of reinforcement and increases the current frequency of all behavior that has been reinforced with food. These two effects occur simultaneously and independently. ... The relation between rate of responding on a variable-interval food schedule and food-deprivation level might be thought of as a model for 
the evocative effect, but it is not an appropriate model because on such a schedule the increased rate is a function of both EO effects. The organism's rate of food-reinforced behavior increases prior [emphasis added] to obtaining any food as the evocative effect of the EO, and may increase further after a response is followed by the more effective food reinforcement. A more appropriate conceptual model for the evocative effect by itself is the direct relation between deprivation level and the initial rate of responding or the total number of responses emitted during extinction. (p. 403)

In effect, Michael makes a clear distinction between increases in behavior that are (a) due to deprivation per se and (b) due to a combination of deprivation and a history of reinforcement with respect to that behavior.

Michael's separation of motivational effects has been criticized by, among others, McDevitt and Fantino (1993), who stated that "the evocative function of an EO seems to bring us no closer to predicting the nature of the response, which is usually dependant upon additional contextual stimuli" (p. 226). Similarly, Klatt and Morris (2001) noted that there may be methodological problems with separating out the valuealtering and behavior-altering effects of MOs because "even though the evocative effect of response deprivation seems to be necessary for the first occurrence of instrumental responding, the effect is often unmeasured" (p. 177).

On balance, perhaps Michael and colleagues are suggesting that the evocative effect occurs at the start of an experimental session before any reinforcer has been delivered within that session. However, this approach seems to ignore the fact that reentering the experimental context at the start of a session will likely possess discriminative properties for the availability of reinforcement, based on previous sessions. In summary, therefore, the evocative function of the EO remains unclear (cf. the value-altering effect) and certainly at the present time seems to remain largely a theoretical rather than an empirical construct.

\section{Lack of Empirical Supporting Evidence}

Perhaps the most serious problem with the concept of MOs is that some aspects of the MO theoretical framework are not strongly rooted in a current and growing body of empirical data. To his credit, Michael (2000) himself admitted that his statements "often go beyond wellestablished empirical support" (p. 402). In addition, previous writings on the MO concept have not incorporated research findings that are typically grouped under the headings of, for example, behavioral economics (Perry \& Fisher, 2001), reinforcement hierarchies (DeLeon et al., 2001), response deprivation (Catania, 1993; Dougher, 1983; Hanley, Iwata, Lindberg, \& Conners, 2003; Klatt \& Morris, 2001; McDevitt \& Fantino, 1993), the Premack principle (Hanley, Iwata, Roscoe, Thompson, \& Lindberg, 2003), and other variables relevant to UEOs (Catania, 1993). It might be argued, therefore, that the MO approach to motivation is too narrow, and thus it has mitigated against the wider application of motivational variables to a wider range of behaviors. 


\section{An Alternative Conceptual Analysis of Motivation}

Given the foregoing criticisms of Michael's treatment of motivation, we have sought to develop an alternative treatment of motivation that derives from Skinner's early work, utilizes only two new terms, does not incorporate the concept of a dual process, and also makes contact with a broader range of existing data.

There has been a tendency within behavior analysis to view most behavior as under the complete control of a discriminative stimulus $\left(\mathrm{S}^{\mathrm{D}}\right)$. However, Skinner (1938) issued a cautionary note concerning the use of the technical term $S^{\mathrm{D}}$ :

[The discriminative stimulus] is perhaps best described as "setting the occasion" for a response. Whether or not the response is to occur does not depend upon the discriminative stimulus, once it is present, but upon other factors. . . Strictly speaking we should refer to a discriminated operant as "occurring in the presence of" rather than "elicited as a response to" $\mathrm{S}^{\mathrm{D}}$. (p. 241)

We will argue that it is these "other factors" that should be regarded as motivational variables. That is, factors that influence the rate of operant responding (i.e., factors that act on two-, three-, four-, or five-term contingencies and so on) but are not part of the discriminated operant contingency are considered motivational.

\section{Consequence-Valuing Operations (CVOs) and Consequence-Valuing Processes (CVPS)}

One methodology for analyzing motivation, based on Skinner's treatment of motivation (Skinner, 1938, 1953), would involve establishing an operant contingency and then determining if the frequency of responding could be manipulated without changing that contingency or the discriminative control over that contingency. Indeed, in a classic study in this area, Skinner fed an animal (that was always 24-hr food deprived) with varying amounts of food just prior to an experimental session. He subsequently observed that response rate during that session decreased as a function of increased supplemental feeding, although the contingency and antecedent stimulus conditions remained unchanged (see Skinner, 1938, p. 393; Figure 1 displays these data).

In colloquial terms, one might view the presession feeding as altering the drive or motivational state inside the animal. In behavior analysis, however, such organocentric terms are usually avoided (see Hineline, 1980; see also Skinner, 1945), and perhaps for this reason, the term establishing operation was first introduced. The EO refers, at least in part, to establishing the reinforcing effectiveness of a particular consequence rather than some internal state.

An alternative term that may be used to describe the type of effect demonstrated by Skinner in 1938 would refer to the effectiveness, or apparent value, of the consequential stimuli that participate in an operant contingency. For this reason, we offer the following two concepts: the consequence-valuing operation (CVO) and the consequence-valuing process (CVP). The former refers to the contextual arrangements made by a researcher or practitioner that are designed to either increase or decrease the value of a consequence in an operant contingency without changing or 


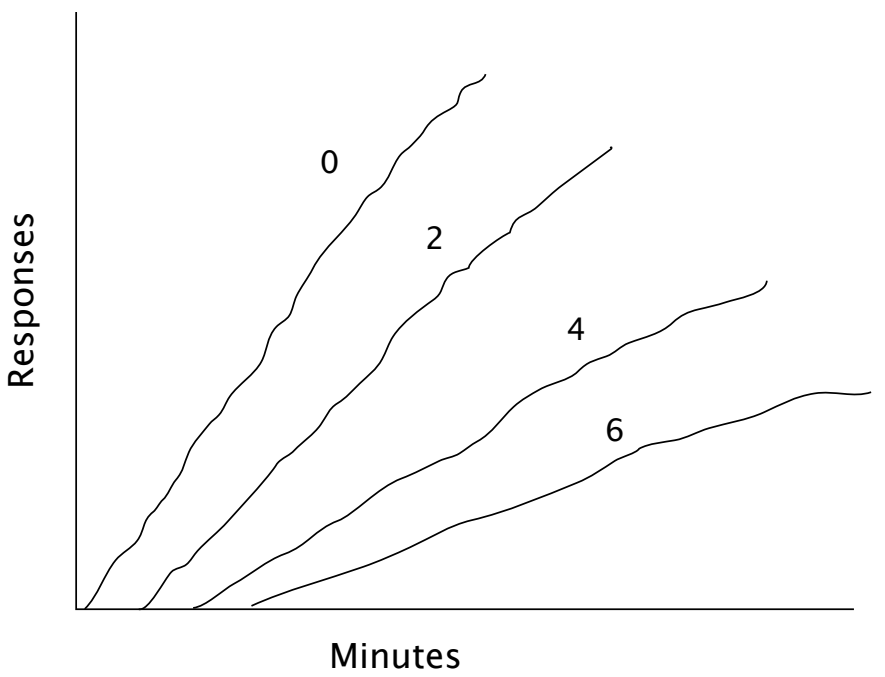

Figure 1. Response rates from Skinner's (1938) study. The numbers above the lines represent food in grams.

altering that contingency, or by demonstrating some interaction between the factors of consequence value and contingency relations. The CVP refers to the behavior changes that occur as a result of the CVO. In Skinner's study, therefore, presession feeding would be defined as a CVO and the changes in response rate that occurred across different prefeeding amounts as a CVP. Of course, Skinner's study provides but one example, so we will work systematically through what we see as the main permutations involved in establishing CVOs and measuring CVPs. In doing so, we will refer to some of the available and relevant applied literature in the area.

Basic operant responding and discriminated operant responding. Table 1 displays a number of functional relations. Row 1 lists the kinds of events necessary to describe basic operant functional relations: a baseline rate of consequence value and a particular stimulus situation $\left(\mathrm{SS}_{1}\right)$ in which at least some kinds of responses are followed by reinforcement. The rate of operant responding can be some arbitrary value above zero, $B$ (indicating baseline). Rows 2 and 3 illustrate what happens when a response is reinforced in some of the elements of a situation but not reinforced in others. Stimuli that are correlated with reinforcement and an increased rate of responding are termed $\mathrm{S}^{\mathrm{D}} \mathrm{s}$, and stimuli that are negatively correlated with reinforcement and correlated with a decreased rate of responding are termed $S^{\Delta} \mathrm{s}$. Thus, Rows 2 and 3 represent discriminative control over responding. The response rate in the presence of the $S^{\mathrm{D}}$ is some value greater than $B$, say $X$, whereas the response rate in the presence of the $S^{\Delta}$ will be some value less than $B$, say $Y$. Figure 2 presents a simplified version of an actual experiment that demonstrated the development of control by a discriminative stimulus and thus represents the functional relations described in Rows 1, 2, and 3. As an aside, we recognize that what we describe as basic operant responding is likely to be under some form of discriminative control, and thus the distinction we have made between basic and discriminated operants is based on the scientist's or practitioner's perspective, rather than the organism's. 


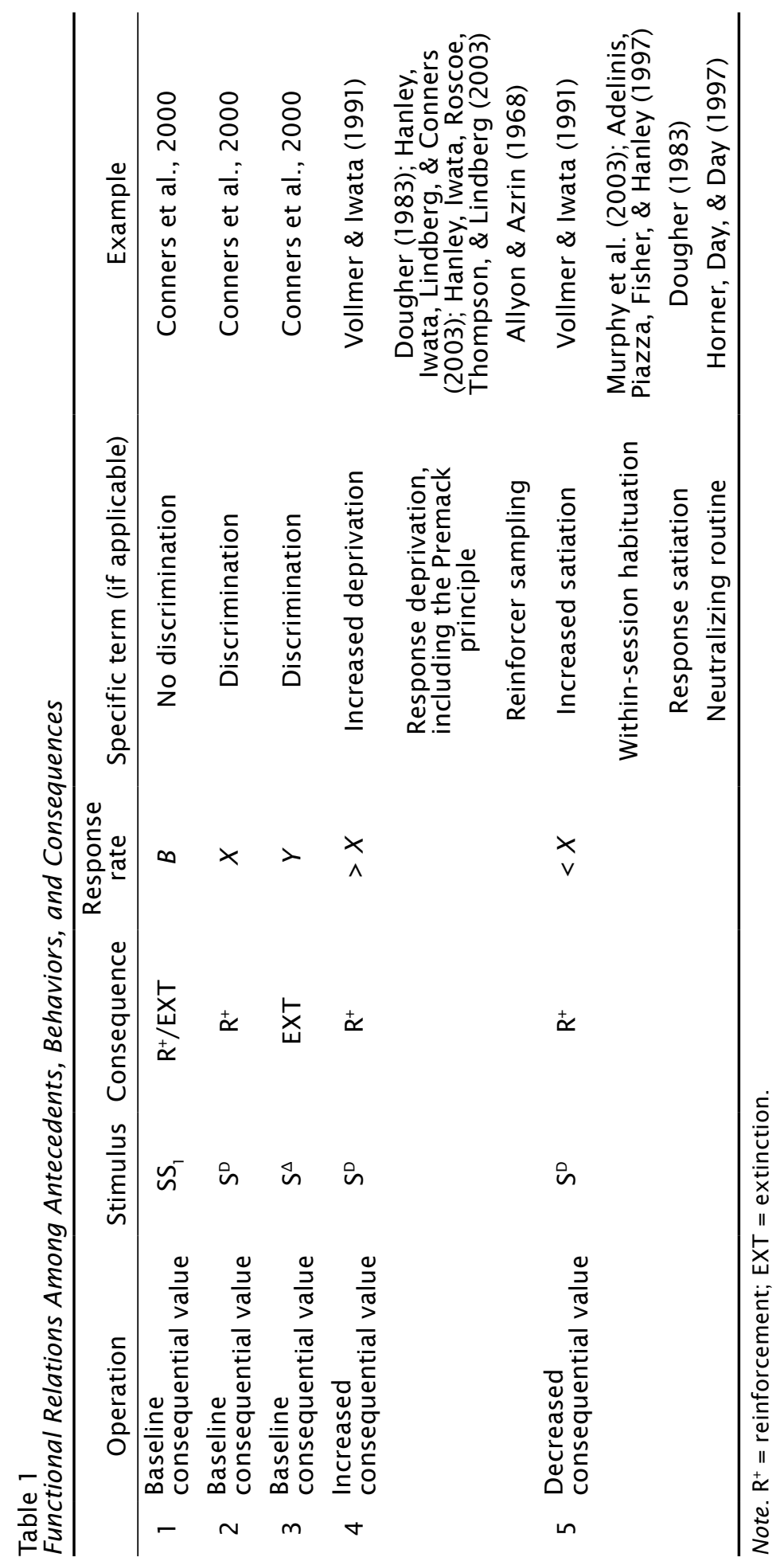




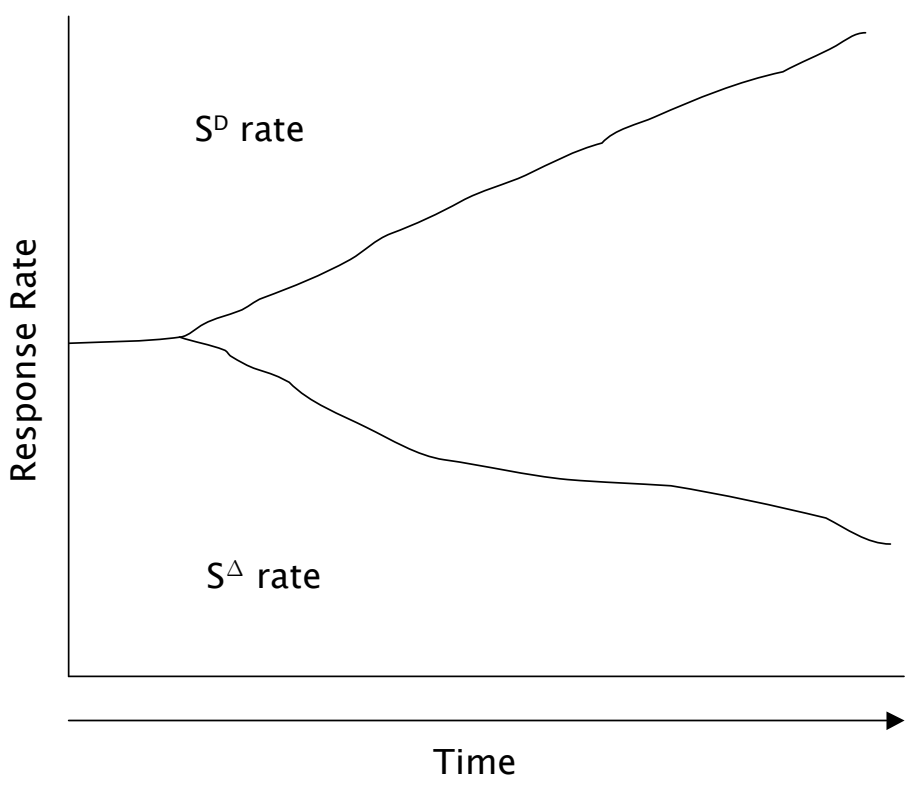

Figure 2. A simplified version of an actual experiment that demonstrated the development of control by a discriminative stimulus.

Increased consequential value. A situation may arise in which a CVO is conducted with the aim of changing response rate. The operation can be deemed to have increased the value of the consequence (i.e., is motivational) if the relations among the events within the n-term discriminated operant remain unchanged. We can speak of a CVP if, as a result of an operation, response rate in the presence of the $S^{\mathrm{D}}$ is increased relative to the baseline response rate in the presence of $S^{D}$ (i.e., greater than $X$ ). Similarly, a CVP has occurred if the response rate in the presence of the $S^{\Delta}$ is increased relative to the response rate in the presence of $S^{\Delta}$ in the baseline condition (i.e., greater than $Y$ ).

An applied example of Row 4 can be seen in Vollmer and Iwata (1991). In this study, CVOs were arranged with respect to food (primary reinforcement), social interaction (conditioned reinforcement), and music (sensory reinforcement). All participants were diagnosed as being profoundly developmentally delayed. In the music condition, for example, a participant who had gone 30 min without musical stimulation was presented with a switch-closing task. This task was consequated with the production of music. All of the response rates were higher in the deprivation than in the baseline condition across all tasks, with one exception. Thus, although the operant contingency remained unchanged, the results of this study indicated that the target responses varied as a function of relative deprivation versus satiation.

Klatt and Morris (2001) noted that response deprivation, which subsumes the Premack principle, could usefully be described as an establishing operation. Hanley, Iwata, Roscoe, et al. (2003) and Hanley, Iwata, Lindberg, et al. (2003) demonstrated that response restriction and the application of the Premack principle could increase the rate of previously nonpreferred activities (i.e., were CVOs). 
An experimenter can also introduce a novel antecedent stimulus into a situation, and leave the n-term contingency unchanged, with the aim of changing consequential value (i.e., a CVO). A CVP has occurred if this consequence-valuing stimulus is correlated with an increase in responding either above $X$ if the $S^{D}$ is present or above $Y$ if the $S^{\Delta}$ is present (Row 4 ). Similar results can be seen when reinforcer sampling is employed. That is, presession exposure to stimuli that share perceptual functions, such as edibles, seems to increase the probability of responding in a given context, relative to a baseline condition (Allyon \& Azrin, 1968).

Decreased consequential value. A similar logic can be applied if some operation is correlated with a response rate that is lower than $X$ in the presence of the $\mathrm{S}^{\mathrm{D}}$ and/or is lower than $Y$ in the presence of the $\mathrm{S}^{\Delta}$. These can be termed consequence-devaluing operations, and Row 5 displays these relationships. Consequence-devaluing processes can be observed, for example, in Vollmer and Iwata (1991). In the satiation condition of Vollmer and Iwata, the participant listened to $30 \mathrm{~min}$ of music prior to exposure to the switch-closing task. The response rate in satiation was always lower than in deprivation across all tasks. Parenthetically, Murphy, McSweeney, Smith, and McComas (2003) have argued that the decrease in reinforcer effectiveness following repeated presentations is due to habituation rather than to satiation. In any case, the decrease in response rate, whether due to satiation or habituation, is a CVP.

Horner, Day, and Day (1997) introduced what they called a neutralizing routine to reduce problem behavior. In the case of one participant, Karl, the problem behavior was aggression or self-injury, or both. An $\mathrm{S}^{\mathrm{D}}$ for this problem behavior was defined as physical interruption when Karl was reaching for a food item on the reinforcer tray. The authors also established that there was an increase in this problem behavior from baseline when Karl had fewer than 5 hr of sleep the previous night. In Karl's case, the devaluing operation (i.e., the neutralizing routine) was the opportunity to take a nap. The probability of the problem behavior for Karl was .295 when the baseline CVO (i.e., fewer than $5 \mathrm{hr}$ of sleep) and the $\mathrm{S}^{\mathrm{D}}$ were in effect. When the consequence-devaluing operation, the neutralizing routine, was introduced, the probability of responding when the $S^{\mathrm{D}}$ was held constant fell to .012. Horner et al. also described a situation in which the probability of responding when the baseline CVO was in effect but the $S^{\mathrm{D}}$ was not present was .167. When the consequence-devaluing operation was arranged, the probability of responding in the absence of the $S^{\mathrm{D}}$ fell to .05.

In all of the above examples, the concepts of CVO and CVP can be readily applied as terms that serve to describe the increase and decrease in the relevant behaviors, independent of other contingencies.

\section{Interactions Between Contingencies and Motivation}

Behavioral economics. Researchers who study behavioral economics have noted that changing the contingencies in an experiment, such that an increase in response rate is needed to produce reinforcement, does not always reliably result in an increased response rate. For example, in Hursh (1978; Experiment 1) response rates increased with increasing variableinterval (VI) schedules. In contrast, in Hursh's Experiment 2, response rates decreased with increasing VI schedules under an identical VI contingency. 
The crucial difference between these experiments was that subjects in Experiment 1 were participating in a closed economy, whereas subjects in Experiment 2 were participating in an open economy. In closed economies, reinforcers are only available within the experimental session; that is, deprivation is not artificially held constant by the experimenter. An open economy is any of a variety of experimental arrangements in which reinforcers are provided outside of the experimental session. The CVO in the case of the Hursh experiments was the between-session feedings, and the CVP was the divergent response rates across both the altered contingencies and altered consequential values.

Perry and Fisher (2001) examined the effects of manipulating both consequential values and contingencies. Their results demonstrated that several variables can interact to influence response allocation between appropriate and destructive behavior. For example, as response effort increased, the higher-quality reinforcer increased in consequential value. Increasing the "price" associated with destructive behavior by adding a punishment procedure canceled out the effects of increasing the response effort and the amount of compliance with requests completed.

Behavioral pharmacology. The administration of drugs can have a considerable influence on behavior even if the operant contingencies are held constant. As such, drugs are examples of CVOs. A common drug administered to individuals with attention-deficit/hyperactivity disorder (ADHD) is methylphenidate (MPH; more commonly known by the brand name Ritalin). It has been difficult, however, to accurately measure the effects of MPH, because most studies have evaluated treatment effects based on participant report, parent report, teacher report, and behavior rating scales, which are often inadequate measures (Stoner, Carey, Ikeda, \& Shinn, 1994). In addition, most studies have relied on between-group design, which may be problematic because there are large individual differences in response to MPH, and dose-response relations are not necessarily linear. Therefore, it is desirable to use single-case designs in order to measure the interaction between operant contingencies and CVOs.

Gulley and Northup (1997) provided a well-controlled example of the effects of MPH on problem behavior, and we take the case of one participant diagnosed with ADHD, Jacob, as an example. Jacob was administered three different dosages-low, medium, and high-and a placebo. Disruptive behavior was significantly reduced at the high dosage, but there were no differences at the low and medium dosages. Interestingly, this pattern of results was almost directly opposite on a reading task: There was no difference between the placebo and the highest dosage, but performance was improved at the lowest dosage. Furthermore, there was no difference in performance on a math task between the placebo and any dosage. The key point to note is that the effects of the CVO were specific to the dosage or area of functioning. In other words, there were complex interactions between behavior and its consequence and the CVO in terms of their effects on the CVP.

\section{Advantages of Using the CVO and CVP Concepts for the Practitioner}

Researchers who manipulate motivational factors have "generally adopted Michael's terminology with respect to [MOs/EOs]" (Laraway et al., 2003, p. 407). Thus, it is incumbent upon us to outline the advantages and 
potential disadvantages of using the conceptual analysis that we have offered, and these are as follows.

The concept of the CVO/CVP is already the de facto approach. Although we have introduced two new terms, we believe that adopting our analysis would require minimal adjustment on the part of practitioners. Indeed, perhaps the concept of the CVO is the de facto approach adopted by applied behavior analysts when they discuss motivational factors. That is, the evocative function of the MO does not seem to play a significant role in behavioral research on motivation. For example, Smith and Iwata (1997) described their criteria for classifying and identifying an antecedent variable as an EO as follows:

Antecedent variables are classified as EOs if, given constant contingencies between behavior and consequences, behavior was altered by the presence or absence of antecedent variables. Thus, when antecedent conditions vary independent of the probability of reinforcement or punishment, behavioral effects are described in terms of EOs. (p. 350)

The approach described by Smith and Iwata for identifying motivational variables seems to overlap substantially with our concept of CVOs and CVPs (also see Valdivia, Luciano, \& Molina, 2006). We have criticized the behavioraltering component of MOs on theoretical grounds, but, perhaps more seriously, the evocative/behavior-altering effect has played virtually no role in any applied study to date. It appears, therefore, that practitioners have already studied motivation without incorporating the dual nature of the MO.

Our analysis is more consistent with behavior-analytic theory and practice. Some researchers have criticized Skinner's approach to motivational factors, claiming that it is inadequate for describing the historical processes that give rise to motivated behavior. For example, Smith and Iwata (1997) claimed that

Skinner's analysis of antecedent events included a functional account of the discriminated operant; however, events such as satiation and deprivation, emotions, aversive stimulation, and other antecedent variables were described in terms of observed stimulus-response relations. Conspicuously absent was an account of the relationship between these antecedent conditions and the consequences that maintain operant behavior. Whereas the discriminative stimulus derives control over responding through a special historical relationship with behavioral consequences, Skinner's account of other antecedents suggests a different source of influence between some antecedent stimuli and behavior. Thus, although no internal contradictions exist in a Skinnerian account of antecedent influences, further theoretical extension is necessary. (p. 346)

The introduction of the terms CVO and CVP is an attempt to provide further theoretical extension of Skinner's account of motivational factors by providing a more precise nomenclature to help clarify the relations among antecedent variables. By focusing on the value of the reinforcer in terms of the level of responding that occurs relative to some previously measured baseline, a CVO approach is clearly consistent with a functional- 
analytic interpretation of behavior-environment interactions. Students and practitioners who are familiar with the concept of stimulus control, and the technical terms $S^{D}$ and $S^{\Delta}$, would likely have little trouble in comprehending and applying a CVO analysis.

More phenomena encompassed results in an increase in scope. In this article, we have described a number of behavioral phenomena that are included in our definition of motivation. In contrast, the MO concept seems to focus on very specific types of relations among variables, and hence many motivational phenomena are not considered. Furthermore, we have not identified any specific relations a priori, but rather have described the pattern of environment-behavior interactions that we think constitute motivational operations and processes. In this manner, perhaps behavior analysts can study motivational variables that traditionally have not been encompassed under the rubric of behavioral psychology (see Catania, 1993, p. 223). For example, the lack of motivation displayed by depressed individuals is clearly an important issue, although the explanations for the processes involved are often rife with explanatory fictions (see Chiesa, 1994). For instance, impaired motivation in depressed patients has been described as a "lack of an appropriate response to explicit reward . . . where depressed patients may not perceive reward as reinforcing because of a low hedonic capacity" (Austin, Mitchell, \& Goodwin, 2000, p. 202). Clearly, the clarity that a parsimonious functional-analytic approach could bring to topics such as this would be useful.

\section{Is There a Loss of Precision?}

The specific subdivisions of the CEO have rarely been employed; rather, the general term EO has been utilized to refer to alterations in motivational factors. This does not necessarily mean that these subdivisions are redundant, and perhaps they could offer more precision and more explanatory power than the concepts of CVO and CVP. In addressing this issue, it is necessary to go into some detail.

Three types of CEOs have been described: a surrogate CEO, which involves correlating a stimulus with a UEO; a reflexive CEO, which involves correlating a stimulus with worsening or improvement; and a transitive CEO, which involves conditional conditioned reinforcement and punishment. In each case, we will argue that the terms CVO and CVP can be combined with existing behavioral terms to adequately describe the range of phenomena described thus far in the applied behavioral literature.

Surrogate CEOs are established when a neutral event is paired with or systematically precedes a UEO or a CEO. In other words, the previously neutral event may acquire the motivational characteristics of the event with which it is paired. For example, correlating a neutral stimulus with painful stimulation may increase the effectiveness of pain reduction as a form of reinforcement and evoke the behavior that has been reinforced with pain reduction. However, the introduction of the term surrogate would seem to be nothing more than a replacement term for the word pairing, and it is questionable if this new term adds any explanatory precision. Furthermore, there are a number of ways in which a previously neutral stimulus can acquire the properties of an unconditioned stimulus, and for which a number of existing modifiers can be applied (see Catania, 1993, for a more 
comprehensive treatment of this issue). For example, a direct contingency might be arranged between the unconditioned and neutral stimulus, or each might participate in the same equivalence class or relational frame (Hayes, Kohlenberg, \& Hayes, 1991; Whelan \& Barnes-Holmes, 2004; Whelan, Barnes-Holmes, \& Dymond, 2006). None of these alternate scenarios would be covered by the term surrogate CEO, although in each case the previously neutral event may acquire the motivational characteristics of the UEO or CEO.

A reflexive CEO is assumed to be established when a stimulus systematically precedes some form of worsening and this worsening does not occur if the stimulus is terminated. For example, a warning stimulus in an avoidance procedure can acquire the capacity to establish its own termination as a form of conditioned reinforcement and can evoke any behavior that has accomplished this termination. However, as McDevitt and Fantino (1993) pointed out, an analysis based on compound stimuli is also plausible. To take an applied example, if a demand were presented (e.g., "Let's start to work in a minute") and these demands were often escaped from, then they can be conceptualized as a reflexive CEO (McGill, 1999). Extending the argument made by McDevitt and Fantino (pp. 226227), an alternative view is that the escape behavior is reinforced because it is correlated with an increase in the time to the task when a demand has been presented but is not correlated with an increase in the absence of the demand. According to McDevitt and Fantino's analysis, the demand and the task together can be considered as a compound $\mathrm{S}^{\mathrm{D}}$ for escape behavior because they are uniquely correlated with an increase in the availability of reinforcement (escape from task) for a particular response (escape behavior). In this case, $\mathrm{S}^{\mathrm{D}}$ and CVO concepts are sufficient to cover this eventuality and, on the grounds of parsimony, are preferable over the extra concept of the reflexive CEO. Indeed, in our view, there must always be some kind of CVO in order for operant responding to occur. The CVO in this example is the baseline rate of reinforcer effectiveness for escaping the task, although it was not systematically manipulated in this case.

Michael (1993a) acknowledged that both a compound stimulus and a CEO approach are possible but disliked the former because the explanation “involving the compound stimulus doesn't seem to isolate the relevant controlling variables as well as the CEO version does" (p. 235). Although Michael argued that the MO approach serves to isolate the relevant controlling variables, it remains the case that both basic and applied researchers have not used his concepts to do so. Once again, therefore, it is possible that many of the subdivisions within the concept of the MO are redundant and the simpler process account provided by CVOs and CVPs, combined with established behavior principles, is sufficient.

Transitive CEOs are established when a certain stimulus condition is correlated with the correlation between another stimulus and some form of worsening or improvement. Thus, the first stimulus establishes the reinforcing or punishing effectiveness of the second stimulus and evokes or suppresses the behavior that has been followed by that reinforcement or punishment. To illustrate our approach, an example from McGill (1999) is presented: 
For example, the instructor's saying “No!" during the activity may momentarily increase the reinforcing effectiveness of escape from the activity and evoke problem behavior that results in escape. If it can be shown that the problem behavior is reinforced by escape from the activity (rather than just from the instructor saying “No!"), then the correction procedure has the properties of a transitive CEO. It is a CEO (rather than an $\mathrm{S}^{\mathrm{D}}$ ) because escape from the activity is equally available whether or not the instructor says "No!" It is transitive in that it operates directly on an independent event (the reinforcing effectiveness of escape from the activity). (p. 409)

The argument for employing the term transitive CEO is that this stimulus acts on an independent event rather than altering its own function. However, almost all antecedent stimuli act on other events rather than altering their own function. Indeed, a surrogate CEO can also act in this way. If, for example, a neutral stimulus correlated with painful stimulation increases the effectiveness of pain reduction as a form of reinforcement and evokes the behavior that has been reinforced with pain reduction, then this can be considered as both a surrogate and transitive CEO. Applying our analysis to the above example, saying the word "no!" would be described as a CVO because the availability of reinforcement (escape from the activity) was not altered. A CVP would be observed when the problem behavior increased following presentation of the word.

In summary, our contention is that the subdivisions of the MO are not necessary because existing behavioral terminology is sufficient, at least for now, to describe motivated behavior with both scope and precision. According to Skinner, "To insist upon the constancy of properties which can be shown not to affect the measurements in hand is to make a fetish of exactitude. ... What is wanted is the 'necessary and sufficient' correlation of a stimulus and a response" (1935/1999, p. 507). In our view therefore, terms such as learned, unlearned, conditioned, unconditioned, first-order, secondorder, and so on can all be employed as modifiers for CVOs, in the same way that we apply these modifiers to antecedent stimuli that are correlated with the differential availability of reinforcement.

\section{Conclusion}

In this article, we criticized the concept of the motivating operation (e.g., Laraway et al., 2003) on the grounds that the terms and concepts employed do not always overlap with traditional behavior-analytic verbal practices, that the dual nature of the MO is unclear, and that there is a lack of adequate contact with empirical data. Instead, we offered two key terms, consequencevaluing operation (CVO) and consequence-valuing process (CVP). In this alternative account, the focus is on the value of the reinforcer in terms of the level of responding that occurs relative to some previously measured baseline, and a distinction between operation and process is incorporated. Our account can accommodate the extant data on motivational factors and consequently is a more parsimonious approach that is no less precise than the MO concept. It is an accepted tenet of the scientific method that if two theories are equal in their explanatory power, then the simpler of the two is superior. We recognize, however, that adopting these terms would require 
some adjustment on the part of the behavioral community. Ultimately, our hope is that the adoption of the CVO and CVP concepts will better facilitate practitioners and researchers in identifying, analyzing, and manipulating target behaviors.

\section{References}

ADELINIS, J. D., PIAZZA, C. C., FISHER, W. W., \& HANLEY, G. P. (1997). The establishing effects of client location on self-injurious behavior. Research in Developmental Disabilities, 18, 383-391.

ALLYON, T., \& AZRIN, N. H. (1968). Reinforcer sampling: A technique for increasing the behavior of mental patients. Journal of Applied Behavior Analysis, 1, 13-20.

AUSTIN, M. P., MITCHELL, P., \& GOODWIN, G. M. (2001). Cognitive defects in depression: Possible implications for neuropathology. British Journal of Psychiatry, 178, 200-206.

CATANIA, C. A. (1992). Learning (3rd ed.). Englewood Cliffs, NJ: Prentice-Hall.

CATANIA, C. A. (1993). Coming to terms with establishing operations. The Behavior Analyst, 16, 219-224.

CHERPAS, C. (1993). Do establishing operations alter reinforcement effectiveness? The Behavior Analyst, 16, 347-349.

CHIESA, M. (1994). Radical behaviorism: The philosophy and the science. Boston, MA: Authors Cooperative.

CONNERS, J., IWATA, B. A., KAHNG, S., HANLEY, G. P., WORSDELL, A. S., \& THOMPSON, R. H. (2000). Differential responding in the presence and absence of discriminative stimuli during multielement functional analyses. Journal of Applied Behavior Analysis, 33, 299-308.

DELEON, I. G., FISHER, W. W., CATTER, V. R., MAGLIERI, K., HERMAN, K., \& MARHEFKA, J. (2001). Examination of relative reinforcement effects of stimuli identified through pretreatment and daily brief preference assessments. Journal of Applied Behavior Analysis, 34, 463-473.

DOUGHER, M. J. (1983). Clinical effects of response deprivation and response satiation procedures. Behavior Therapy, 14, 286-298.

GULLEY, V., \& NORTHUP, J. (1997). Comprehensive school-based behavioral assessment of the effects of methylphenidate. Journal of Applied Behavior Analysis, 30, 627-638.

HANLEY, G. P., IWATA, B. A., LINDBERG, J. S., \& CONNERS, J. (2003). Responserestriction analysis: I. Assessment of activity preferences. Journal of Applied Behavior Analysis, 36, 47-58.

HANLEY, G. P., IWATA, B. A., ROSCOE, E. M., THOMPSON, R. H., \& LINDBERG, J. S. (2003). Response-restriction analysis: II. Alteration of activity preferences. Journal of Applied Behavior Analysis, 36, 59-76.

HAYES, S. C., KOHLENBERG, B. S., \& HAYES, L. J. (1991). The transfer of specific and general consequential functions through simple and conditional equivalence relations. Journal of the Experimental Analysis of Behavior, 56, 119-137.

HINELINE, P. N. (1980). The language of behavior analysis: Its community, its functions, and its limitations. Behaviorism, 8, 67-86.

HORNER, R. H., DAY, M. H., \& DAY, J. R. (1997). Using neutralizing routines to reduce problem behaviors. Journal of Applied Behavior Analysis, 30, 601-613. 
HURSH, S. R. (1978). The economics of daily consumption controlling foodand water-reinforced responding. Journal of the Experimental Analysis of Behavior, 29, 475-491.

IWATA, B. A., SMITH, R. G., \& MICHAEL, J. L. (2000). Current research on the influence of establishing operations on behavior in applied settings. Journal of Applied Behavior Analysis, 33, 411-418.

KELLER, F. S., \& SCHOENFELD, W. N. (1950). Principles of psychology. New York: Appleton-Century-Crofts.

KLATT, K. P., \& MORRIS, E. K. (2001). The Premack principle, response deprivation, and establishing operations. The Behavior Analyst, 24, 173-180.

LARAWAY, S., SNYCERSKI, S., MICHAEL, J., \& POLING, A. (2001/2002). The abative effect: A new term to describe the action of antecedents that reduce operant responding. The Analysis of Verbal Behavior, 18, 101-104.

LARAWAY, S., SNYCERSKI, S., MICHAEL, J., \& POLING, A. (2003). Motivating operations and terms to describe them: Some further refinements. Journal of Applied Behavior Analysis, 36, 407-414.

LEIGLAND, S. (1984). On "setting events" and related concepts. The Behavior Analyst, 7, 41-45.

MCDEVITT, M. A., \& FANTINO, E. (1993). Establishing operations and the discriminative stimulus. The Behavior Analyst, 16, 225-227.

MCGILL, P. (1999). Establishing operations: Implications for the assessment, treatment, and prevention of problem behavior. Journal of Applied Behavior Analysis, 32, 393-418.

MCPHERSON, A., \& OSBORNE, J. G. (1988). Control of behavior by an establishing stimulus. Journal of the Experimental Analysis of Behavior, $49,213-227$.

MICHAEL, J. (1982). Distinguishing between discriminative and motivational functions of stimuli. Journal of the Experimental Analysis of Behavior, 37, $149-155$.

MICHAEL, J. (1983). Evocative and repertoire-altering effects of an environmental event. The Analysis of Verbal Behavior, 2, 19-21.

MICHAEL, J. (1988). Establishing operations and the mand. The Analysis of Verbal Behavior, 2, 19-21.

MICHAEL, J. (1993a). Establishing operations. The Behavior Analyst, 16, 191-206.

MICHAEL, J. (1993b). Concepts and principles of behavior analysis. Kalamazoo, MI: Society for the Advancement of Behavior Analysis.

MICHAEL, J. (2000). Implications and refinements of the establishing operation concept. Journal of Applied Behavior Analysis, 33, 401-410.

MICHAEL, J., HIXSON, M. D., \& CLARK, J. W. (1997). The role of motivation in the S-R issue. Journal of the Experimental Analysis of Behavior, 67, 239-241.

MURPHY, E. S., MCSWEENEY, F. K., SMITH, R. G., \& MCCOMAS, J. J. (2003). Dynamic changes in reinforcer effectiveness: Theoretical, methodological, and practical implications for applied research. Journal of Applied Behavior Analysis, 36, 421-438.

PERRY, A.C., \& FISHER, W. W. (2001). Behavioral economic influences on treatments designed to decrease destructive behavior. Journal of Applied Behavior Analysis, 34, 211-215.

SKINNER, B. F. (1938). The behavior of organisms: An experimental analysis. Oxford: Appleton-Century. 
SKINNER, B. F. (1945). The operational analysis of psychological terms. Psychological Review, 52, 270-277.

SKINNER, B. F. (1953). Science and human behavior. New York: Macmillan.

SKINNER, B. F. (1999). The genetic nature of the concepts of stimulus and response. In Cumulative record: Definitive edition (pp. 504-524). Acton, MA: Copley. (Original work published 1935)

SMITH, R. G., \& IWATA, B. A. (1997). Antecedent influences on behavior disorders. Journal of Applied Behavior Analysis, 30, 343-375.

STONER, G., CAREY, S., IKEDA, M., \& SHINN, M. (1994). The utility of curriculum-based measurement for evaluating the effects of methylphenidate on academic performance. Journal of Applied Behavior Analysis, 27, 101-113.

VALDIVIA, S., LUCIANO, C., \& MOLINA, F. J. (2006). Verbal regulation of motivational states. The Psychological Record, 56, 577-595.

VOLLMER, T. R., \& IWATA, B. A. (1991). Establishing operations and reinforcement effects. Journal of Applied Behavior Analysis, 24, 279-291.

WHELAN, R., \& BARNES-HOLMES, D. (2004). The transformation of consequential functions in accordance with the relational frames of Same and Opposite. Journal of the Experimental Analysis of Behavior, 82, 177-195.

WHELAN, R., BARNES-HOLMES, D., \& DYMOND, S. (2006). The transformation of consequential functions in accordance with the relational frames of more-than and less-than. Journal of the Experimental Analysis of Behavior, 86, 317-335. 